Canadian Oncology

Nursing Journal

Revue canadienne

de soins infirmiers

en oncologie

Volume 31, Issue 1 • Winter 2021

elSSN: 2368-8076 


\title{
Instrument de mesure de la nausée chez l'enfant : traduction française et validité des visages pour les patients canadiens francophones en oncopédiatrie
}

\author{
par Anne Choquette, Araby Sivananthan, Annie Guillemette, Erin O’Shaubhnessy, Martha Pinheiro-Maltez, Linda MacKeigan, \\ Anne-Marie Langevin et L. Lee Dupuis
}

\section{INTRODUCTION}

L es nausées et les vomissements induits par la chimiothé-rapie (NVIC) nuisent à la qualité de vie tant des adultes que des patients pédiatriques atteints d'un cancer (Dupuis, Milne-Wren, Cassidy et al., 2010; Farrell, Brearley, Pilling et Molassiotic, 2013; Russo, Cinausero, Gerratana et al., 2014; Hinds, Gattuso, Billups et al., 2009; Sommariva, Pongiglione, et Tarricone, 2016). Les vomissements et les haut-le-cœur sont des symptômes qui s'évaluent objectivement, alors que la nausée est subjective et plus difficile à mesurer. En général, l'intensité de la nausée chez les adultes peut être décrite à l'aide d'échelles d'évaluation visuelle analogique ou qualitative. Il existe à cette fin des instruments validés et recommandés par des spécialistes du domaine, comme celui de la Multinational Association of Supportive Care in Cancer (en ligne: www.mascc. org) (Hesketh, Gralla, du Bois et Tonato, 2016).

En 2006, nous avons élaboré le Pediatric Nausea Assessment Tool (PeNAT [instrument de mesure de la nausée chez l'enfant]), une échelle visuelle analogique à quatre visages dérivée de l'échelle de la douleur de Wong-Baker, qui s'accompagne d'un scénario explicatif pour l'évaluation de la nausée chez

\section{AUTEURES}

Anne Choquette, inf. aut., M.Sc.inf., CPON, Hôpital de Montréal pour enfants, Centre hospitalier de l'Université McGill, Montréal (Québec)

Araby Sivananthan, B.Sc. Institut de recherche, Hospital for Sick Children, Toronto (Ontario)

Annie Guillemette, M.Sc., Hôpital de Montréal pour enfants, Centre hospitalier de l'Université McGill, Montréal (Québec)

Erin O'Shaughnessy, inf. aut., M.Sc.inf., Centre hospitalier pour enfants de l'est de l'Ontario, Ottawa (Ontario)

Martha Pinheiro-Maltez, inf. aut., M.Sc.inf., CPHON, Centre hospitalier pour enfants de l'est de l'Ontario, Ottawa (Ontario)

Linda MacKeigan, Ph.D., Faculté de pharmacie Leslie Dan, Université de Toronto, Toronto (Ontario)

Anne-Marie Langevin, M.D., UT Health Science Center, San Antonio, Texas

L. Lee Dupuis, pharmacienne agréee., Ph.D., FCSHP, Institut de recherche, Hospital for Sick Children, Toronto (Ontario); Faculté de pharmacie Leslie Dan, Université de Toronto, Toronto (Ontario); Département de pharmacie, Hospital for Sick Children, Toronto (Ontario)

Correspondance : L. Lee Dupuis pharmacienne agréée, Ph.D., 555 University Ave., Toronto (Ontario) M5M 1X3

lee.dupuis@sickkids.ca

Tél. : 416-813-7654, poste 309355 ; Téléc. : 416-813-5979

DOI:10.5737/236880763117382 les patients anglophones âgés de 4 à 18 ans sous chimiothérapie (Dupuis, Taddio, Kerr et al., 2006). Le PeNAT anglais, valide et fiable (Dupuis, Taddio, Kerr et al., 2006), est utilisé pour décrire l'intensité de la nausée chez les enfants recevant différents traitements chimiothérapiques (Vol, Flank, Lavoratore et al., 2016; Patel, Lavoratore, Flank et al., 2019; Clinton, Dowling et Capra, 2012), pour jauger l'efficacité des interventions visant à soulager les nausées et vomissements (Dupuis, Kelly, Krischer et al., 2018; Evans, Malvar, Garretson et al., 2018) et pour évaluer la gravité des nausées dans le cadre des soins cliniques habituels (McKionnon et Jupp, 2019). Cependant, son utilisation se limite aux patients anglophones.

Au Canada, il y a deux langues officielles : le français et l'anglais. Dans le recensement de 2016, 20 \% des répondants ont indiqué que le français était la langue la plus parlée à la maison (Statistique Canada, 2016), un pourcentage équivalent à plus de 7 millions de Canadiens. Ainsi, s'il n'a pas accès à un outil écrit dans sa langue, l'enfant francophone pourra difficilement exprimer l'intensité de ses nausées. Il devient donc impossible d'évaluer l'intensité des symptômes avec précision et la persistance des nausées entravera la qualité de vie (Dupuis, Lu, Mitchell et al., 2016). De plus, sans outil en français pour évaluer la gravité des nausées, les enfants francophones ne peuvent pas participer aux études sur les traitements antiémétiques. Nous avons donc entrepris de traduire le PeNAT de l'anglais au français et de tester la validité des visages pour établir l'intensité des nausées et vomissements induits par chimiothérapie (NVIC) chez les patients canadiens francophones en oncopédiatrie.

\section{MÉTHODOLOGIE}

L'étude s'est faite en deux étapes. Premièrement, le PeNAT et les documents connexes (scénario explicatif, journal du patient et fiche d'instructions du journal) ont été traduits en français puis révisés. Deuxièmement, on a testé la validité des visages et des pièces justificatives en français auprès des parents et des patients en pédiatrie. Pendant la première étape, les professionnels de la santé du Centre hospitalier pour enfants de l'est de l'Ontario (CHEO) ont révisé la version française du PeNAT. Durant la deuxième étape, on a recruté patients et parents à l'Hospital for Sick Children (SickKids) et à l'Hôpital de Montréal pour enfants (MCH [Montreal Children's Hospital]). Létude a reçu l'approbation des comités d'éthique de la recherche du SickKids, du MCH, du CHEO et de l'Université McGill. Tous les participants ont donné leur assentiment ou leur consentement éclairé, le cas échéant. 


\section{Traduction française du PeNAT}

L PeNAT et les documents connexes en anglais, préalablement validés (Dupuis, Taddio, Kerr et al., 2006), ont été traduits en français par un cabinet de traduction professionnel. Le processus de traduction respectait les normes actuelles (Office des normes générales du Canada/Canadian General Standards Board, 2008) ainsi que les pratiques exemplaires (Txabarriaga, 2009) et s'est fait en étapes : 1) traduction des documents par un linguiste professionnel; 2) vérification, par un deuxième linguiste professionnel, de la concordance de la traduction avec les documents sources; 3) contrôle de la qualité pour vérifier la sémantique, la grammaire, la syntaxe, la ponctuation et le niveau de lecture; 4) révision par un troisième linguiste pour que la traduction soit complète et fluide. Le cabinet de traduction a tenu compte des commentaires des quatre chercheuses francophones (A-ML, EO, MP-M, LLD) dans sa révision des documents traduits.

Par la suite, le PeNAT et les documents connexes en français ont été révisés par trois professionnels de la santé bilingues du domaine de l'oncopédiatrie pour s'assurer que les versions traduites respectaient le contenu des documents anglais, qu'elles seraient comprises par les répondants, et qu'elles permettraient de recueillir l'information souhaitée. Les professionnels de la santé bilingues ont d'abord lu les documents originaux et les traductions françaises selon un ordre de présentation aléatoire (anglais vs français et vice versa). Ils ont ensuite répondu à un questionnaire structuré (annexe 1) lors d'un entretien en personne pendant lequel les chercheurs discutaient de toutes les modifications suggérées. Puis, le cabinet de traduction a révisé les traductions en tenant compte de ces commentaires. Lorsque les corrections étaient importantes, les textes étaient soumis à une deuxième ronde de révision par des professionnels de la santé bilingues. À chaque étape du processus, les chercheuses révisaient les documents français afin d'en assurer la cohérence avec les documents anglais.

\section{Test de validité des visages}

Les patients admissibles étaient âgés de 4 à 18 ans, ne souffraient d'aucune déficience physique ou cognitive empêchant l'utilisation du PeNAT et devaient recevoir, comme patients hospitalisés ou en consultation externe, une chimiothérapie modérément ou hautement émétogène (Dupuis, Boodham, Sung et al., 2011) au SickKids ou au MCH. De plus, les patients admissibles parlaient français à la maison. On a recruté les participants en consultant les listes de patients hospitalisés et les horaires de consultations cliniques. Pour que des enfants de différents âges soient représentés, on a procédé à un échantillonnage dirigé. Un échantillon de sept à dix participants est suffisant pour déterminer la compréhensibilité (Willis, 2005). Ainsi, nous avons enrôlé des patients et leurs parents jusqu'à ce que 10 participants consécutifs ne proposent aucune révision de fond à la dernière version de l'échelle française et la jugent facile ou très facile d'utilisation. Les patients étaient recrutés par groupes de cinq. Chaque patient n'a participé qu'une seule fois et a reçu une carte-cadeau en guise de remerciement à la fin l'étude.
Trois jours après le dernier bloc de chimiothérapie des patients participant à l'étude, les parents ont répondu à un questionnaire standardisé (annexe 2) administré par l'équipe de recherche, par téléphone ou en personne. Ce questionnaire réunissait des questions sur le PeNAT et sur les documents connexes en français en mettant l'accent sur la facilité d'utilisation, la lisibilité et la clarté pour mesurer l'intensité des nausées. La première partie, axée sur le PeNAT, comportait quatre questions auxquelles il fallait répondre par oui ou non et trois questions demandant des suggestions pour améliorer le PeNAT. L'autre partie du questionnaire portait sur le journal et consistait en quatre questions ouvertes pour comprendre les points forts de l'outil, recueillir des suggestions d'amélioration, et demander aux parents d'évaluer la facilité ou la difficulté d'utilisation du journal sur une échelle en cinq points (de très facile à très difficile). Il fallait environ dix minutes pour remplir le questionnaire, et les patients pouvaient choisir d'y participer ou non.

Par la suite, les chercheuses ont passé en revue les réponses du premier groupe de cinq participants et décidé si une révision s'imposait. Le recrutement était interrompu jusqu'à ce que cette décision soit prise. Les versions révisées du PeNAT et des documents connexes en français ont été relues par des professionnels de la santé bilingues, comme mentionné précédemment. À la dernière étape, les chercheuses ont révisé les documents finaux en français pour s'assurer de leur cohérence et de leur concordance avec les versions anglaises.

\section{Utilisation du PeNAT et des pièces justificatives}

L'équipe de recherche a enseigné à chaque participant comment utiliser le PeNAT et les documents connexes en français, puis observé la première utilisation par le patient, qui était invité à utiliser l'outil français pour évaluer l'intensité de la nausée au moins deux fois par jour pendant la phase aiguë de son prochain bloc de chimiothérapie modérément ou hautement émétogène. On a également demandé aux patients ou aux parents de noter dans le journal français l'heure de chaque vomissement et administration d'antiémétiques pendant cette période. Dans sa définition, la phase aiguë commençait à la première dose du bloc de chimiothérapie et se terminait 24 heures après la dernière dose du même bloc. Le bloc de chimiothérapie correspondait à la période de jours consécutifs pendant laquelle le traitement est administré. Les parents devaient remettre les journaux remplis aux chercheuses à leur prochaine visite à l'hôpital.

\section{Collecte de données}

Les caractéristiques démographiques ainsi que les renseignements sur la chimiothérapie et les médicaments antiémétiques du patient ont été recueillis dans son dossier médical. Les journaux donnaient de l'information sur l'intensité des nausées, le contrôle des vomissements et l'administration des antiémétiques.

\section{Analyse}

Les réponses des parents au questionnaire ont été colligées à des fins d'examen. La validité des visages a été jugée avérée lorsque dix participants consécutifs ont indiqué que les 
documents connexes en français étaient faciles ou très faciles à utiliser et n'ont proposé aucune modification du PeNAT en français.

Les données démographiques et le niveau de soulagement des NVIC en phase aiguë rapporté par les patients ont été analysés à l'aide de statistiques descriptives. Pour que le soulagement des NVIC en phase aiguë soit considéré comme complet, il devait y avoir absence d'épisodes de nausées et de vomissements (score PeNAT maximum : 1). Le soulagement partiel des NVIC en phase aiguë correspondait à un ou deux épisodes de vomissements en 24 heures ou un score PeNAT maximum de 2. Enfin, le soulagement des NVIC en phase aiguë était un échec lorsqu'il se produisait plus de deux épisodes de vomissements en 24 heures, ou un score PeNAT maximum de 3 ou 4.

\section{RÉSULTATS}

\section{Traduction française du PeNAT}

De l'avis des chercheuses francophones, la première traduction française du PeNAT et des documents connexes était trop familière. Ils l'ont donc retournée aux traducteurs médicaux pour révision. Par contre, la deuxième version était acceptable. Deux groupes de trois professionnels de la santé bilingues ayant trois ans ou plus d'expérience en oncopédiatrie (cinq infirmières autorisées et un pharmacien) au CHEO ont ensuite proposé quelques changements. Selon l'un des relecteurs du premier groupe, le terme français « hautle-cœur » utilisé dans le journal pour traduire « retching » n'était pas clair. Après discussion entre les chercheuses francophones et consultation du Grand dictionnaire terminologique de l'Office québécois de la langue française (http:// www.granddictionnaire.com/index.aspx) et des linguistes professionnels, ce terme a été remplacé par « effort pour vomir ». Les documents français révisés ont ensuite été relus par le deuxième groupe de professionnels de la santé, qui n'a suggéré aucune autre modification. Les commentaires des groupes de professionnels de la santé sont résumés au tableau 1.

\section{Test de validité des visages}

Deux cohortes de cinq patients ont été recrutées au MCH. Les caractéristiques démographiques et l'information sur les traitements sont présentées au tableau 2. Les parents ont dit que leur enfant était capable d'utiliser le PeNAT pour décrire sa nausée, et ils trouvaient le journal facile ou très facile à remplir. Trois parents ont proposé d'utiliser des visages

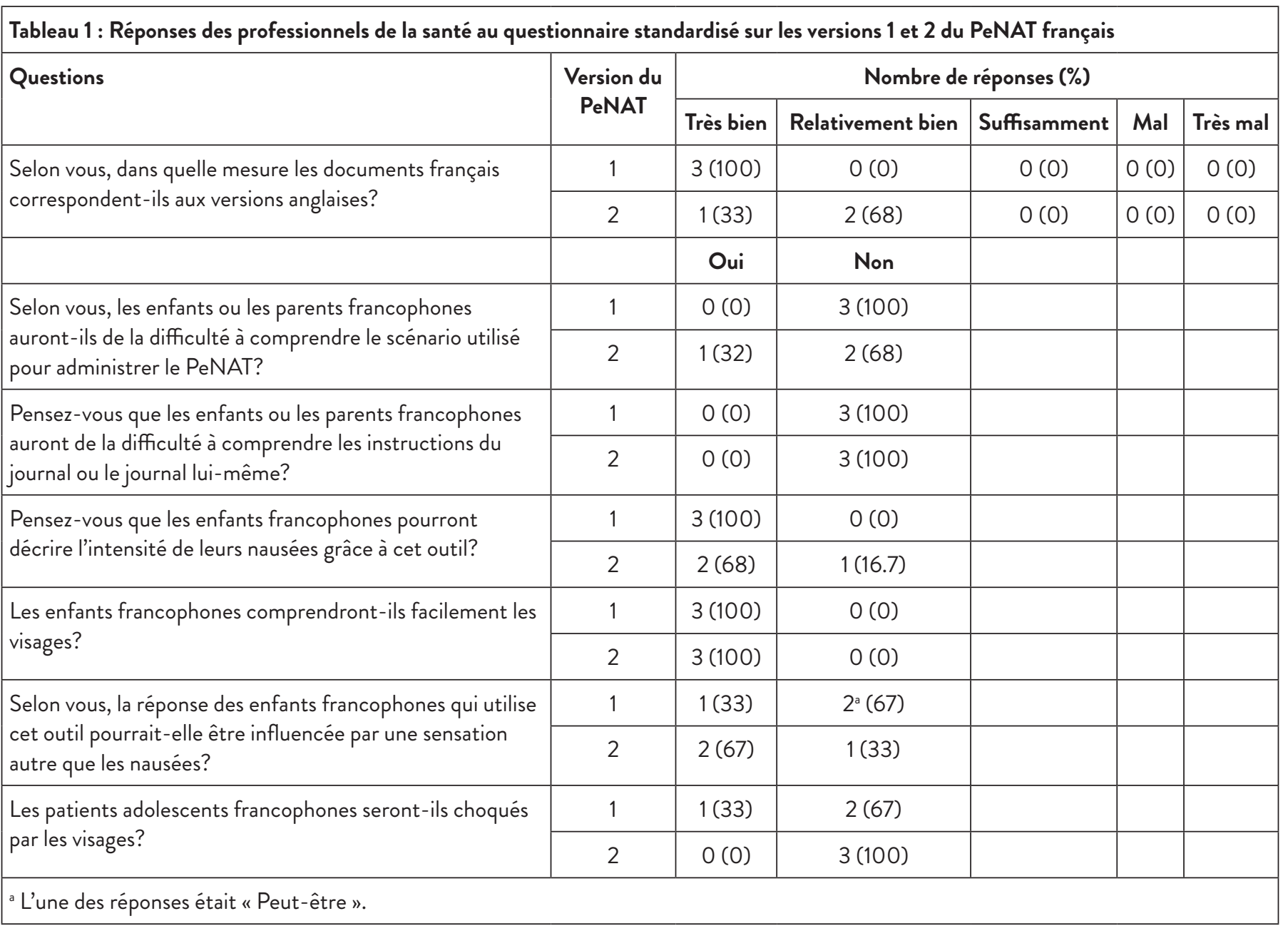




\begin{tabular}{|c|c|c|c|}
\hline Caractéristiques & $\begin{array}{l}\text { Première cohorte } \\
\qquad(N=5)\end{array}$ & $\begin{array}{l}\text { Deuxième cohorte } \\
\quad(N=5)\end{array}$ & $\begin{array}{c}\text { Tous les patients } \\
(N=10)\end{array}$ \\
\hline \multicolumn{4}{|l|}{ Patients } \\
\hline \multicolumn{4}{|l|}{$\hat{A} g e$} \\
\hline 4 à 8 ans (nombre; \%) & $1(20)$ & $4(80)$ & $5(50)$ \\
\hline 8 à 18 ans (nombre; \%) & $4(80)$ & $1(20)$ & $5(50)$ \\
\hline Âge médian (écart) & $10(7-14)$ & $7(5-15)$ & $7,5(5-15)$ \\
\hline \multicolumn{4}{|l|}{ Diagnostic de cancer } \\
\hline Leucémie lymphoblastique aiguë & $1(20)$ & $4(80)$ & $5(50)$ \\
\hline Autre $^{*}$ & $4(80)$ & $1(20)$ & $5(50)$ \\
\hline \multicolumn{4}{|l|}{ Traitement } \\
\hline \multicolumn{4}{|l|}{ Chimiothérapie hautement émétogène } \\
\hline Bléomycine, cyclophosphamide, doxorubicine, étoposide + vincristine & 0 & $1(20)$ & $1(10)$ \\
\hline Brentuximab, cyclophosphamide, doxorubicine étoposide & $1(20)$ & 0 & $1(10)$ \\
\hline Cyclophosphamide + cytarabine & 0 & $2(40)$ & $2(20)$ \\
\hline Étoposide + ifosfamide & $1(20)$ & 0 & $1(10)$ \\
\hline \multicolumn{4}{|l|}{ Chimiothérapie modérément émétogène } \\
\hline Cytarabine, méthotrexate + rituximab & $1(20)$ & 0 & $1(10)$ \\
\hline Doxorubicine + vincristine & $1(20)$ & $1(20)$ & $2(20)$ \\
\hline Erwinia L-asparaginase + méthotrexate & 0 & $1(20)$ & $1(10)$ \\
\hline Méthotrexate + vincristine & $1(20)$ & 0 & $1(10)$ \\
\hline \multicolumn{4}{|l|}{ Prophylaxie antiémétique administrée } \\
\hline Ondansétron & $1(20)$ & $4(80)$ & $5(50)$ \\
\hline Ondansétron + aprépitant & $1(20)$ & $1(20)$ & $2(20)$ \\
\hline Ondansétron + dexaméthasone & $2(40)$ & 0 & $2(20)$ \\
\hline Ondansétron + dimenhydrinate & $1(20)$ & 0 & $1(10)$ \\
\hline
\end{tabular}

\begin{tabular}{|l|c|c|c|c|}
\hline Tableau 3 : Réponses des parents aux questions sur leur expérience de l'utilisation du PeNAT français et du journal avec leur enfant \\
\hline \multirow{2}{*}{ Questions } & Réponses & \multicolumn{3}{|c|}{ Nombre de réponses des parents } \\
\cline { 3 - 5 } & & Première cohorte & Deuxième cohorte & Tous les parents \\
\hline $\begin{array}{l}\text { Pensez-vous que j'ai suffisamment expliqué comment utiliser } \\
\text { l'outil pour que votre enfant soit vraiment capable de décrire sa } \\
\text { nausée? }\end{array}$ & Oui & 5 & 5 & 10 \\
\cline { 3 - 5 } & & 0 & 0 & 0 \\
\hline $\begin{array}{l}\text { Croyez-vous que votre enfant a pu décrire le degré de nausée } \\
\text { qu'il/elle ressentait à l'aide de cet outil? }\end{array}$ & Oui & 5 & 5 & 10 \\
\cline { 2 - 5 } & Non & 0 & 0 & 0 \\
\hline Pour votre enfant, était-ce facile pour de choisir le visage qui & Oui & 4 & 5 & 9 \\
\cline { 2 - 5 } correspondait à ce qu'il/elle ressentait? & Non & 1 & 0 & 1 \\
\hline Pensez-vous que votre enfant a été influencé par autre chose & Oui & 2 & 0 & 2 \\
\cline { 2 - 5 } que la nausée lors de l'utilisation de l'outil? & Non & 3 & 5 & 8 \\
\hline $\begin{array}{l}\text { Dans l'ensemble, évaluez la facilité ou la difficulté d'utilisation } \\
\text { du journal }\end{array}$ & Très facile & 1 & 4 & 5 \\
\cline { 2 - 5 } & Facile & 4 & 1 & 5 \\
\hline
\end{tabular}


colorés plutôt que des images en noir et blanc dans le PeNAT. Deux adolescents ont suggéré d'utiliser une autre échelle de visages pour leur groupe d'âge. Un parent a rapporté que, pour son enfant, l'intensité des nausées se situait entre deux visages. Aucune modification n'a été apportée aux documents du PeNAT français à la lumière de ces commentaires (voir les versions finales à l'annexe 3).

\section{Utilisation du PeNAT et des documents connexes}

En moyenne, les patients ont rempli quatre pages du journal des NVIC (médiane de 3,5; écart de 2 à 8 ). Au total, les 10 patients ont remis 95 scores individuels (moyenne de 9,5 par patient; écart de 3 à 21). Les scores du PeNAT vont de 1 à 4 et les valeurs maximales rapportées par patient se situent entre 2 et 4 (médiane de 2,5). Tous les patients ont fait état de nausées (score PeNAT $\geq 2$ ) au moins une fois pendant la phase aiguë. En phase aiguë, aucun patient n'a été complètement soulagé des NVIC. Par contre, quatre patients ont ressenti un soulagement partiel et le contrôle des NVIC a échoué pour six autres patients.

\section{DISCUSSION}

Le PeNAT est un outil valide et fiable pour évaluer l'intensité des nausées des patients anglophones en oncopédiatrie (Dupuis, Tddio et al., 2006). La présente étude confirme la validité des visages de la version française du PENAT pour les enfants et adolescents francophones du Canada traités par chimiothérapie. Tous les parents ont dit que leur enfant était capable d'utiliser le PeNAT pour communiquer l'intensité de ses nausées et que le PeNAT et les pièces justificatives étaient faciles ou très faciles à utiliser.

Les antiémétiques recommandés dans les lignes directrices de la pratique clinique sont efficaces pour prévenir les vomissements induits par la chimiothérapie (Hesketh, Bohlke, Lyman et al., 2015; Patel, Robinson, Thackry et al., 2017; Gilmore, Peacock, Gu et al., 2014). Cependant, ils sont moins efficaces pour soulager les nausées (Vol et al., 2016; Patel, Lavoratore et al., 2020; Sparavalo, Hagen, Chong et al., 2012). Nous avons besoin d'outils validés pour évaluer l'intensité des nausées et ainsi déterminer l'efficacité des interventions visant à soulager les nausées et les vomissements chez les patients en pédiatrie. Jusqu'à tout récemment, de tels outils faisaient défaut pour les enfants sous chimiothérapie. Par conséquent, l'évaluation des interventions de contrôle des NVIC chez les enfants a été franchement entravée. Le PeNAT a tout d'abord été conçu et validé pour les patients anglophones en pédiatrie. Pour qu'il soit largement appliqué tant dans la recherche qu'en clinique, il doit être traduit dans d'autres langues. Nous avons choisi d'en produire une version en français, l'une des langues officielles du Canada, et d'évaluer la validité des visages auprès des enfants francophones canadiens. La validité des visages constitue la première étape vers le test de validité exhaustif.

Chez nos patients, les nausées étaient mal maîtrisées, ce qui fait écho à d'autres études évaluant l'intensité des nausées chez les enfants à l'aide d'un outil validé (Patel, Lavoratore et al., 2019; Dupuis, Kelly et al., 2018; Flank, Sparavalo, Vol et al., 2017; Flank, Nadeem, Moledina et al., 2017). Pour arriver à soulager complètement les NVIC, il peut être nécessaire de prescrire des antiémétiques spécifiques en plus des antiémétiques administrés pour prévenir les vomissements induits par la chimiothérapie. Le PeNAT aidera les professionnels de la santé et les parents à mieux comprendre la lourdeur des symptômes des patients en oncopédiatrie. Par exemple, l'utilisation systématique $\mathrm{du}$ PeNAT auprès des patients pédiatriques recevant une chimiothérapie permettrait de savoir pour quels patients il conviendrait d'ajuster la prophylaxie antiémétique pour mieux contrôler les nausées.

Le respect des normes internationales en matière de traduction médicale et la participation à la fois des patients en oncopédiatrie et de leurs parents aux tests de validité des visages constituent les points forts de la présente étude, qui est toutefois limitée par le fait qu’elle n’a été menée que dans un seul pays. Les visages présentés dans notre version française du PeNAT pourraient ne pas être valides pour les patients en oncopédiatrie des autres nations francophones. Autre limite : les tests de validité du PeNAT français sont incomplets. Les prochaines études devront explorer d'autres formes de validité telles que la validité prédictive et la validité conceptuelle.

En résumé, nous avons traduit le PeNAT et les pièces justificatives en français et établi la validité des visages du PeNAT auprès des enfants francophones canadiens recevant une chimiothérapie anticancéreuse, ce qui facilitera leur participation à de futurs essais cliniques multicentriques d'interventions visant à soulager les NVIC. De plus, l'utilisation du PeNAT français comme outil clinique pour optimiser le contrôle des NVIC des enfants francophones pourra maintenant faire l'objet de recherches qui poursuivront le processus de validation des versions française et anglaise du PeNAT.

\section{REMERCIEMENTS}

Nous remercions les patients en pédiatrie et leurs parents qui ont généreusement participé à l'étude. Nous souhaitons également remercier les professionnels de la santé du Centre hospitalier pour enfants de l'est de l'Ontario pour la révision du document et des versions préliminaires, Daryl Moczygemba pour ses conseils, Ana Olteanu et Ashlee Vennettilli pour leur aide concernant les procédures de l'étude, et Navpreet Kaur pour le soutien administratif.

\section{FINANCEMENT}

La présente étude a été financée par le Pediatric Oncology Group of Ontario Research Unit.

\section{CONFLIT D'INTÉRÊTS}

Les auteurs n'ont aucun conflit d'intérêts à divulguer. 


\section{RÉFÉRENCES}

Canadian General Standards Board. (2008). Translation Services. Gatineau: Standards Council of Canada.

Clinton, F., Dowling, M., \& Capra, M. (2012). An audit of chemotherapy-induced nausea and vomiting in children. Nursing Children and Young People, 24, 18-23.

Dupuis, L. L., Boodhan, S., Sung, L., Portwine, C., Hain, R., McCarthy, P., Holdsworth, M., \& Pediatric Oncology Group of Ontario (2011). Guideline for the classification of the acute emetogenic potential of antineoplastic medication in pediatric cancer patients. Pediatr Blood Cancer, 57, 191-8.

Dupuis, L. L., Kelly, K. M., Krischer, J. P., Langevin, A. M., Tamura, R. N., Xu, P., Chen, L., Kolb, E. A., Ullrich, N. J., Sahler, O., Hendershot, E., Stratton, A., Sung, L., \& McLean, T. W. (2018). Acupressure does not improve chemotherapy-induced nausea control in pediatric patients receiving highly emetogenic chemotherapy: A single blinded, randomized, controlled trial. Cancer, 124, 1188-96.

Dupuis, L. L., Lu, X., Mitchell, H. R., Sung, L., Devidas, M., Mattano, L. A., Jr, Carroll, W. L., Winick, N., Hunger, S. P., Maloney, K. W., \& Kadan-Lottick, N. S. (2016). Anxiety, pain, and nausea during treatment of standard risk childhood acute lymphoblastic leukemia: A prospective, longitudinal study from the Children's Oncology Group. Cancer, 122, 1116-25.

Dupuis, L. L., Milne-Wren, C., Cassidy, M., Barrera, M., Portwine, C., Johnston, D. L., Silva, M. P., Sibbald, C., Leaker, M., Routh, S., \& Sung, L. (2010). Symptom assessment in children receiving cancer therapy: The parents' perspective. Support Care Cancer, 18, 281-99.

Dupuis, L. L., Taddio, A., Kerr, E. N., Kelly, A. \& MacKeigan, L. (2006). Development and validation of a pediatric nausea assessment tool (PeNAT) for use by children receiving antineoplastic agents. Pharmacotherapy, 26, 1221-31.

Evans, A., Malvar, J., Garretson, C., Kolovos, E. P., \& Baron Nelson, M. (2018). The use of aromatherapy to reduce chemotherapy-induced nausea in children with cancer: A randomized, double-blind, placebo-controlled trial. Journal of Pediatric Oncology Nursing, 35, $392-8$.

Farrell, C., Brearley, S., Pilling, M., \& Molassiotis, A. (2013).The impact of chemotherapy-related nausea on patients' nutritional status, psychological distress and quality of life. Support Care Cancer, 21, 59-66.

Flank, J., Nadeem, K., Moledina, S., et al. (2017). Nausea and vomiting in children and adolescents receiving intrathecal methotrexate: A prospective, observational study. Pediatr Blood Cancer, 64, e26603.

Flank, J., Sparavalo, J., Vol, H., et al. (2017). The burden of chemotherapy-induced nausea and vomiting in children receiving hematopoietic stem cell transplantation conditioning: A prospective study. Bone Marrow Transplantation, 52, 1294-9.

Gilmore, J., Peacock, N., Gu, A., et al. (2014). Antiemetic guideline consistency and incidence of chemotherapy-induced nausea and vomiting in US community oncology practice: INSPIRE Study. Journal of Oncology Practice, 10, 68-74.

Hesketh, P., Bohlke, K., Lyman, G. H. et al. (2015). Antiemetics: American Society of Clinical Oncology focused guideline update. Journal of Clinical Oncology, 34(4), 381-6. https://doi.org/10.1200/ JCO.2015.64.3635.

Hesketh, P. J., Gralla, R. J., du Bois, A., \& Tonato, M. (1998). Methodology of antiemetic trials: Response assessment, evaluation of new agents and definition of chemotherapy emetogenicity. Support Care Cancer, 6, 221-7.

Hinds, P., Gattuso, J., Billups, C., et al. (2009). Aggressive treatment of non-metastatic osteosarcoma improves health-related quality of life in children and adolescents. European Journal of Cancer, 45, 2007-14.

McKinnon, K. \& Jupp, J. (2019). Integration of a nausea and vomiting assessment tool into antineoplastic management of pediatric oncology patients. J Oncol Pharm Pract. https://doi. org/10.1177/1078155219892666

Patel, P., Lavoratore, S. R., Flank, J., et al. (2020). Chemotherapyinduced nausea and vomiting control in pediatric patients receiving ifosfamide plus etoposide: a prospective, observational study. Support Care Cancer, 28(2), 933-938.

https://doi.org/10.1007/s00520-019-04903-0

Patel, P., Robinson, P., Thackray, J., et al. (2017). Guideline for the prevention of acute chemotherapy-induced nausea and vomiting in pediatric cancer patients: A focused update. Pediatr Blood Cancer, 64, e26542.

Russo, S., Cinausero, M., Gerratana, L., et al. (2014). Factors affecting patient's perception of anticancer treatments side-effects: An observational study. Expert opinion on drug safety, 13, 139-50.

Sommariva, S., Pongiglione, B., \& Tarricone, R. (2016). Impact of chemotherapy-induced nausea and vomiting on health-related quality of life and resource utilization: A systematic review. Critical reviews in oncolog $\gamma /$ hematology, 99, 13-36.

Sparavalo, J., Haggen, L., Chong, D., et al. (2012). Prevalence of chemotherapy-induced nausea and vomiting in children receiving hematopoietic stem cell transplantation conditioning. The Hospital for Sick Children.

Statistics Canada. (2016) Language Highlight Tables, 2016 Census. Government of Canada.

Txabarriaga, R. (2009). IMIA Guide on Medical Translation. International Medical Interpreters Association. https://www. imiaweb.org/

Vol, H., Flank, J., Lavoratore, S., et al. (2016). Poor chemotherapyinduced nausea and vomiting control in children receiving intermediate or high-dose methotrexate. Support Care Cancer, 24, 1365-71.

Willis, G. (2005). Cognitive interviewing: A tool for improving questionnaire design. Sage. 


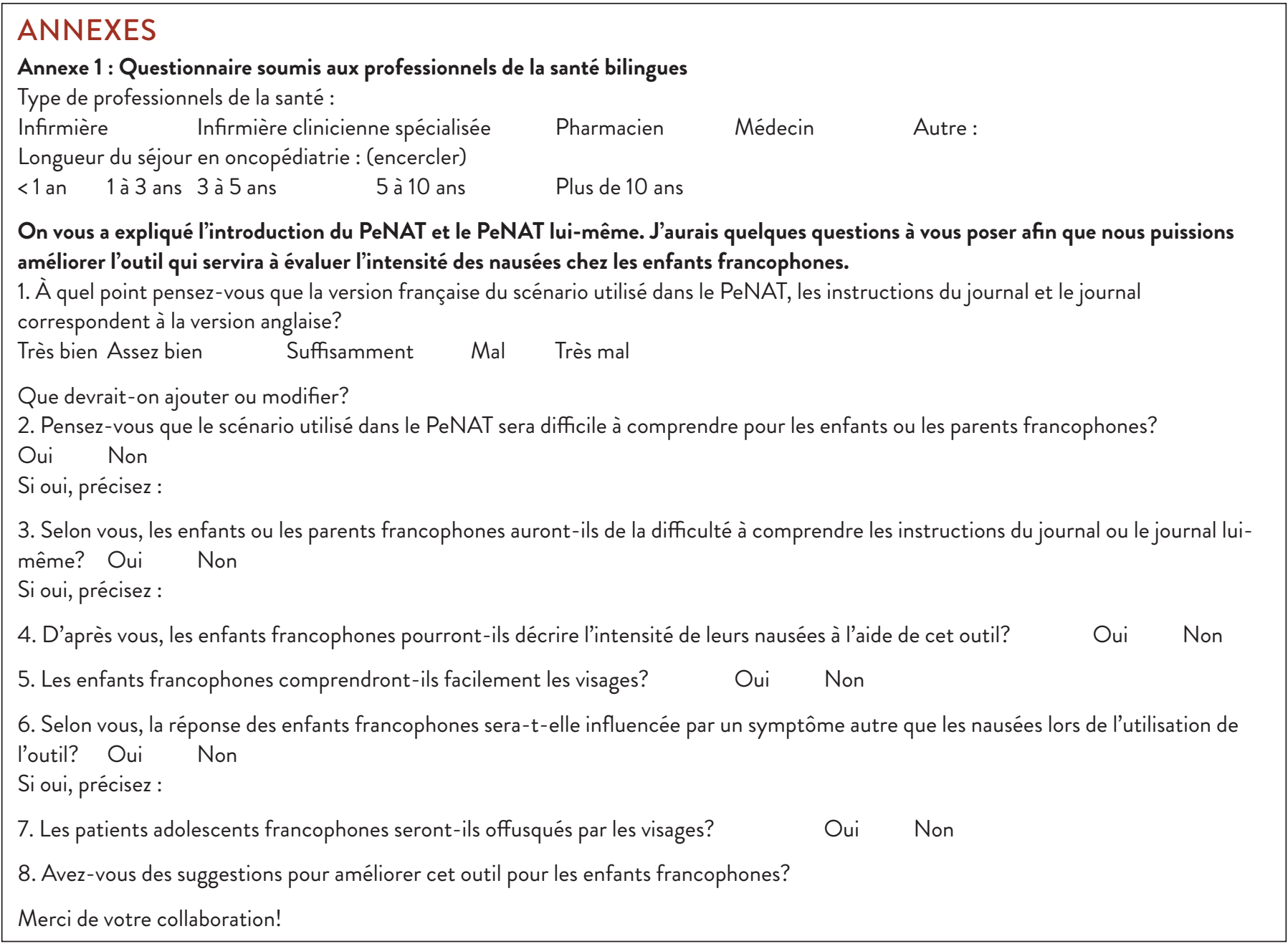




\section{Annexe 2 : Questionnaire soumis aux participants}

Votre enfant vient juste d'évaluer l'intensité de ses nausées à l'aide du PeNAT. Puis-je vous poser quelques questions sur le processus? Vos réponses nous aideront à améliorer le PeNAT. Le questionnaire vous prendra environ 5 minutes.

\section{PeNAT}

1. Pensez-vous que j'ai suffisamment décrit comment utiliser l'outil pour que votre enfant soit capable de bien décrire ses nausées?

2. Croyez-vous que votre enfant a pu me dire le degré de nausée qu'il/elle ressentait à l'aide de cet outil?

3. Était-ce facile pour votre l'enfant de choisir le visage qui correspondait à ce qu'il/elle ressentait?

3. Pensez-vous que votre enfant a été influencé par un symptôme autre que la nausée lors de l'utilisation de l'outil?

4. Si oui, quel était cet autre symptôme? Dans quelle mesure pensez-vous qu'il a influencé les réponses de votre enfant?

5. Selon vous, de quelle autre manière pourrions-nous améliorer la formulation des phrases dans le PeNAT?

6. Auriez-vous d'autres suggestions à faire au sujet du PeNAT?

\section{Journal des NVIC}

1. Y a-t-il quelque chose dans le journal que vous avez trouvé confus ou difficile à comprendre?

2. Qu'avez-vous particulièrement aimé dans le journal?

3. Selon vous, de quelle manière pourrait-on améliorer la formulation des phrases dans le journal?

4. Dans l'ensemble, évaluez la facilité ou la difficulté d'utilisation du journal en cochant l'une des cases ci-dessous.

$\square$ Très difficile

$\square$ Difficile

$\square$ Ni trop difficile ni trop facile

$\square$ Facile

$\square$ Très facile

5. Auriez-vous d'autres suggestions à faire au sujet du journal des NVIC?

Merci de votre collaboration! 
Annexe 3 : PeNAT et documents connexes en français

\subsection{Scripts pour l'administration du PeNAT} SCÉNARIO D'ADMINISTRATION DU PeNAT (outil

d'évaluation des nausées chez l'enfant)

Pour les enfants âgés de 4 à 8 ans :

As-tu déjà vomi (ou autre terme employé par la famille)?

Si tu as déjà vomi, as-tu eu mal au ventre juste avant de vomir (ou autre terme employé par la famille)? À quel point?

Cette sensation s'appelle la nausée ou se sentir nauséeux. Dans ta famille, vous appelez cette sensation

Si tu n'as jamais vomi, as-tu déjà senti que tu allais vomir (ou autre terme utilisé par la famille), mais que tu ne l'as pas fait?

Si tu as déjà senti que tu allais vomir, as-tu eu mal au ventre à ce moment-là? Cette sensation s'appelle la nausée ou

se sentir nauséeux. Dans ta famille, vous appelez cette sensation

Certains enfants qui ont des traitements de chimio se sentent nauséeux (ou autre terme employé par la famille) et d'autres non. En ce moment, à quel enfant ressembles-tu le plus?

Si l'enfant répond qu'il n'éprouve aucune nausée, montrez-lui les visages 1 et 2 .

Certains enfants qui ont des traitements de chimio ne ressentent aucune nausée (ou autre terme employé par la famille), comme pour ce visage, et d'autres ressentent un peu de nausée (ou autre terme employé par la famille), comme pour ce visage-ci. [Pointez du doigt chacun des visages ainsi que le mot descriptif correspondant (aucune et peu) au moment approprié.]

En ce moment, à quel enfant ressembles-tu le plus?

Si l'enfant répond qu'il éprouve une certaine nausée, montrez-lui les visages 3 et 4 .

Certains enfants qui ont des traitements de chimio ressentent de la nausée (ou autre terme employé par la famille), comme pour ce visage, et d'autres ressentent beaucoup de nausées (ou autre terme employé par la famille), comme pour ce visage-ci. [Pointez du doigt chacun des visages ainsi que le mot descriptif correspondant au moment approprié.]

En ce moment, à quel enfant ressembles-tu le plus?

Pour les enfants âgés de 8 ans et plus :

As-tu déjà vomi (ou autre terme employé par la famille)?

Si tu as déjà vomi, as-tu eu mal au ventre (ou à l'estomac) juste avant de vomir (ou autre terme employé par la famille)? À quel point? Cette sensation s'appelle la nausée ou se sentir nauséeux. Dans ta famille, vous appelez cela

Si tu n'as jamais vomi, as-tu déjà senti que tu allais vomir (ou autre terme employé par la famille), mais que tu ne l'as pas fait?

Si tu as déjà senti que tu allais vomir, as-tu eu mal au ventre (ou à l'estomac) à ce moment-là? _________-_. Cette sensation s'appelle la nausée ou se sentir nauséeux. Dans ta famille, vous appelez cela

Certains enfants qui ont des traitements de chimio se sentent nauséeux (ou autre terme employé par la famille) et d'autres non. Ces visages représentent des enfants qui ne ressentent aucune nausée, des enfants qui en ressentent un peu, d'autres qui en ressentent plus et enfin, d'autres qui en ressentent énormément. [Pointez du doigt chaque visage au moment approprié.] En ce moment, quel visage te représente le mieux?

\section{SCÉNARIO EXPLICATIF POUR L'UTILISATION DU JOURNAL}

Pour les enfants âgés de 4 à 8 ans. Le scénario vise le parent ou le tuteur.

Voici une feuille d'instructions et un journal [montrez les feuilles distinctes]. Nous vous donnerons une feuille " Journal du patient " pour chaque jour où vous participez à l'étude. Sur chaque feuille, la date de la journée est indiquée [montrez la date dans le haut de chaque feuille].

Nausée

À l'aide des visages, nous aimerions que vous aidiez à évaluer l'intensité des nausées qu'éprouve votre enfant au lever, au coucher, de même que chaque fois que votre enfant se sent nauséeux.

Afin que votre enfant évalue ses nausées:

1. Dites à votre enfant : "Certains enfants qui ont des traitements de chimio se sentent nauséeux (ou autre terme employé par la famille), et d'autres non. En ce moment, à quel enfant ressembles-tu le plus?"

Si votre enfant répond qu'il n'éprouve aucune nausée, montrez-lui les visages 1 et 2 . $\rightarrow$ Passez à l'étape 2 .

Si votre enfant répond qu'il éprouve une certaine nausée, montrez-lui les visages 3 et 4 . $\rightarrow$ Passez à l'étape 3 .

2. Montrez les visages 1 et 2 à votre enfant. Dites-lui : « Certains enfants qui ont des traitements de chimio n'ont pas de nausées (ou autre terme employé par la famille), comme pour ce visage-ci, et d'autres ont un peu de nausées (ou autre terme employé par la famille), comme pour celui-ci. À quel enfant ressembles-tu le plus en ce moment? " [Pointez du doigt chacun des visages ainsi que le mot descriptif correspondant au moment approprié.]

3. Montrez les visages 3 et 4 à votre enfant. Dites-lui : " Certains enfants qui ont des traitements de chimio ressentent une certaine nausée (ou autre terme employé par la famille), comme pour ce visage, et d'autres ont beaucoup de nausées (ou autre terme employé par la famille), comme pour ce visage-ci. À quel enfant ressembles-tu le plus en ce moment? " [Pointez du doigt chacun des visages ainsi que le mot descriptif correspondant.]

4. Cochez la case qui représente le mieux comment votre enfant se sent. Notez l'heure à laquelle votre enfant a évalué son degré de nausée.

\section{Vomissements}

Au cours de l'étude, indiquez toutes les fois où votre enfant vomit ou qu'il éprouve de la nausée. Si chaque épisode de vomissements ou de nausée survient à un intervalle de plus d'une minute, veuillez noter dans la case l'heure à laquelle cela se produit [pointez du doigt la case appropriée]. 
Médicaments

Indiquez le nom des médicaments que votre enfant prend contre la nausée et les vomissements. Pour chaque jour où votre enfant prend ses médicaments contre la nausée et les vomissements, veuillez écrire dans le journal le nom du médicament et l'heure à laquelle votre enfant l'a pris [pointez du doigt les cases appropriées dans le journal]. Sur la feuille d'instructions se trouve une liste de médicaments contre la nausée et les vomissements [montrez la liste et vérifiez si le patient reçoit certains de ces médicaments].

\section{SCÉNARIO EXPLICATIF SUR LA FAÇON D'UTILISER LE JOURNAL}

Pour les enfants âgés de 9 ans et plus. Le scénario s'adresse à l'enfant et au parent ou au tuteur.

Voici une feuille d'instructions et un journal [montrez chacune des feuilles]. Nous vous donnerons une feuille "Journal du patient " pour chaque jour où vous participez à l'étude. Sur chaque feuille, la date de la journée où nous aimerions que vous la remplissiez est indiquée [montrez la date dans le haut de chaque feuille].

Nausée

À l'aide des visages, nous aimerions que vous aidiez à évaluer l'intensité de la nausée qu'éprouve votre enfant au lever, au coucher, de même que chaque fois que votre enfant se sent nauséeux.

Afin que votre enfant évalue sa nausée, demandez-lui :

"Certains enfants qui ont des traitements de chimio se sentent nauséeux (ou autre terme employé par la famille), et d'autres non. Ces visages représentent des enfants qui ne ressentent aucune nausée, des enfants qui ressentent un peu de nausées, d'autres qui en ressentent encore plus et enfin, d'autres qui en ressentent énormément. Quel visage te représente le mieux en ce moment? " [Pointez du doigt chaque visage au moment approprié.]

Cochez la case qui représente le mieux comment votre enfant se sent. Notez l'heure à laquelle votre enfant a évalué sa nausée.

\section{Vomissements}

Pendant ta participation à l'étude, indique toutes les fois que tu vomis ou que tu éprouves de la nausée. Si chaque vomissement ou nausée est espacé de plus d'une minute, écris dans la case l'heure à laquelle cela arrive [pointez du doigt la case appropriée].

\section{Médicaments}

Indique le nom des médicaments que tu prends contre la nausée et les vomissements. Pour chaque journée au cours de laquelle tu as pris des médicaments contre la nausée et les vomissements, écris dans le journal le nom de ces médicaments et l'heure à laquelle tu les as pris [montrez les cases appropriées dans le journal]. Sur la feuille d'instructions, il y a une liste de médicaments contre la nausée et les vomissements [montrez la liste et vérifiez si le patient reçoit certains d'entre eux].
Journal du patient

Instructions pour le journal du patient

\section{Fiche d'instructions}

Étude sur la nausée et les vomissements induits par la chimiothérapie

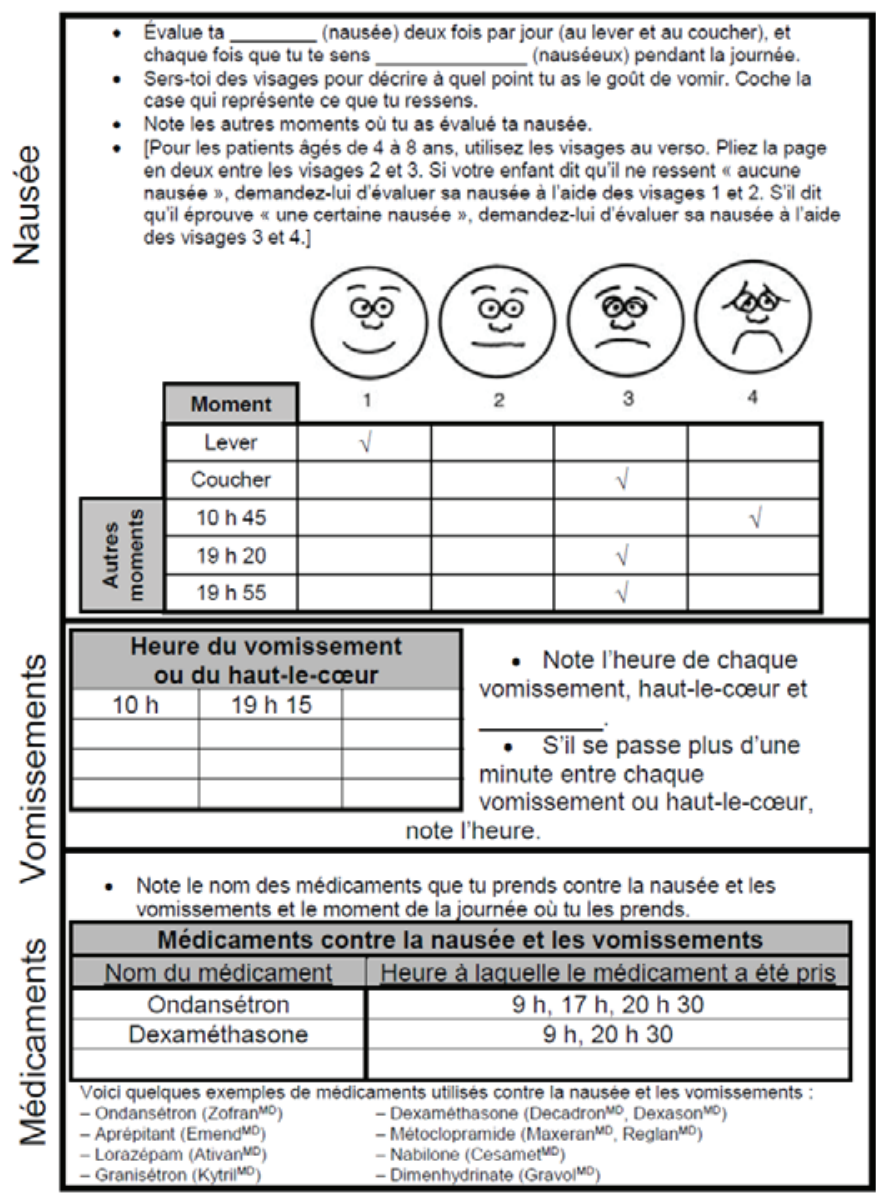

